\title{
Simulation on Denoising Method for Fiber Optic Gyroscope Signal
}

\author{
Ping Sun, Zhi-Lu Zhang, Xiang-Jin Wang, Zhe Li \\ Department of Optoelectronic Equipment, Wuhan Ordnance N. C. O. Academy, China \\ E-mail:sp_9046@163.com
}

\begin{abstract}
Fiber Optics Gyroscopes (FOGs) are widely used due to their low cost. This paper proposes a simulation on denoising method for FOG signal. Hybrid Kalman filter algorithm are applied to denoise the FOG signal in both static and dynamic conditions. Single axis and three axis FOG signals with different angular rates are used in the simulations. Adaptive moving average technique is used to detect the discontinuities in order to select proper Kalman filter gain parameters. The performance is validated by Allan variance analysis and compared with single KF filter. Results show that the amplitudes of rate random walk noise are reduced by 10 times.
\end{abstract}

Keywords-FOG; hybrid kalman filter; denoising

\section{INTRODUCTION}

Recently, the inertial navigation systems (INS) are widely used in land vehicles such as self-propelled howitzers, Infantry fighting vehicles and tanks. Fiber optics gyroscopes (FOGs) are preferred due to their low cost and comparable accuracy [1]. The angular rate in static condition and in dynamic condition is very different due to optics and electronics associated with the FOG.

FOG angular rate signals describe a random process of object movement [2]. For static condition, the Allan variance is a technique for quantifying different noise coefficients [3]. In dynamic condition of FOG, bias drift and response rate are the measured metrics for denoising algorithm. This paper proposes a simulation on denoising method for FOG signal. In literature, FOG static signal denoising algorithms are discussed widely. For the Different signal processing techniques like Discrete Wavelet Transform (DWT) [4] and Kalman filter (KF) [5]. The aim of this paper is to simulate an algorithm [6] which can choose proper KF parameters that will denoise both the static and dynamic condition FOG signal. Improvement in case of the FOGs dynamic condition can be seen in the simulation results. In real time dynamic condition, the noise level varies in different regions as well as different angular rotations of FOG. The implementation of the real time simulation is schemed. The rest of the paper is organized as follows: Section two presents hybrid Kalman filter for denoising FOG signal, Section three briefs the implementation of the real time simulation method, and Section four presents simulation results followed by conclusions in Section five.

\section{ALGORITHM OF HYBRID KALMAN FILTER}

Inertial navigation systems often use Kalman filter for signal filtering. This section presents detailed information of the hybrid Kalman filter which will be used in the simulation. Usually, Kalman filter can be represented by the following equations [2].

$$
\hat{x}_{k}^{-}=A \hat{x}_{k-1}+B u_{k}
$$

$$
P_{k}^{-}=A P_{k-1} A^{T}+Q_{k}
$$

$$
\begin{gathered}
K_{k}=P_{k}^{-} H^{T}\left(H P_{k}^{-} H^{T}+R_{k}\right)^{-1} \\
\hat{x}_{k}=\hat{x}_{k}^{-}+K_{k}\left(z_{k}-H \hat{x}_{k}^{-}\right) \\
P_{k}=\left(I-K_{k} H\right) P_{k}^{-}
\end{gathered}
$$

where $\mathrm{xk}$ is the state vector at time $\mathrm{k}$, uk is the optional control input at time $\mathrm{k}, \mathrm{Qk}$ is the process noise at time $\mathrm{k}, \mathrm{Rk}$ is the measurement noise at time $\mathrm{k}, \mathrm{zk}$ is the measurement taken at time $\mathrm{k}, \mathrm{Pk}$ is error covariance matrix at time $\mathrm{k}, \mathrm{A}, \mathrm{B}$ and $\mathrm{H}$ are state space representation matrices.

In order to denoise the system effectively, we should give appropriate initial values to $\mathrm{Rk}$, as well as $\mathrm{Qk}$ and $\mathrm{P} 0$. The range of KF gain varies between 0 and 1 . The measurement noise and the process noise can be identified with static signal analysis. Allan variance analysis can be used as a method to evaluation the reduction of noise. We assume that the process noise bandwidth is located at a range of lower frequencies, and the bandwidth of the measurement noise is at higher frequency range.

For input data $\mathrm{x}$, we adapt a moving average window of length $2 \mathrm{q}+1$ :

$$
y_{i}=\frac{1}{2 q+1} \sum_{j=-q}^{q} x_{i+j} \text {, }
$$


where $y$ is the output data.

An adaptive filter can be obtained after some iterations of the above process [7].

By using the adaptive moving average technique for $2 q+1$ number of samples, a new signal $\mathrm{Yt}$ is as follows:

$$
Y_{t}=\frac{1}{q_{H}(t)+q_{T}(t)} \sum_{i=-q_{T}(t)}^{q_{H}(t)} X_{t+i}
$$

where, $\mathrm{q}$ is the parameter used in simple moving average.

\section{IMPLEMENTATION OF REAL TIME SIMULATION METHOD}

For a time-varying purpose, the system is adapted as follows:

$$
\widehat{x}_{k+1 / k}=A_{k} \widehat{x}_{k / k-1}+D_{k}+L_{k}\left(y_{k}-C_{k} \widehat{x}_{k / k-1}\right) \text {, }
$$

with arbitrary chosen initial condition:

$$
x_{0 /-1}=m_{0}
$$

Lk is the Kalman gain matrix:

$$
L_{k}=A_{k} P_{k} C_{k}^{T}\left(C_{k} P_{k} C_{k}^{T}+R_{k}\right)^{-1}
$$

The covariance error

$$
P_{k}=\operatorname{cov}\left(x_{x}-\hat{x}_{k / k-1}\right)
$$

is recursively computed through the discrete periodic equation:

$$
\begin{aligned}
P_{k+1} & =A_{k} P_{k} A_{k}^{T}+Q_{k} \\
& -A_{k} P_{k} C_{k}^{T}\left(C_{k} P_{k} C_{k}^{T}+R_{k}\right)^{-1} C_{k} P_{k} A_{k}^{T},
\end{aligned}
$$

where,

$$
P_{0}=\operatorname{cov}\left(x_{0 /-1}\right)
$$

is a freely chosen definite positive initial condition [8]. The detail implementation steps of the proposed algorithm are in Fig. 1.

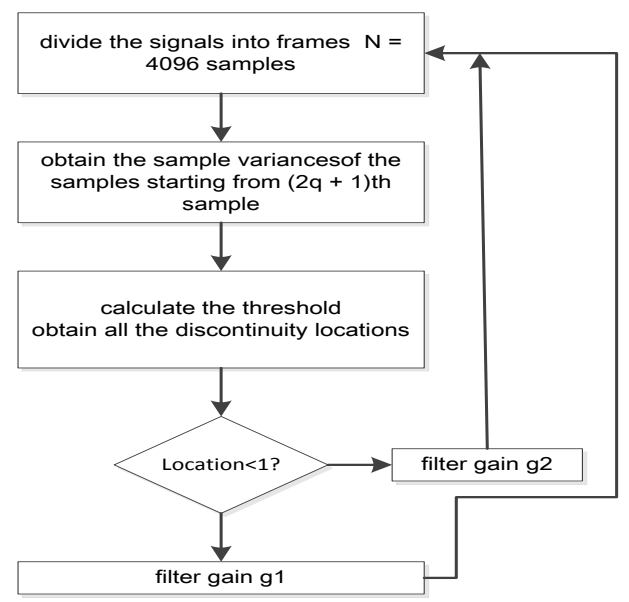

Figure 1. Gyroscope KF filtering algorithm.
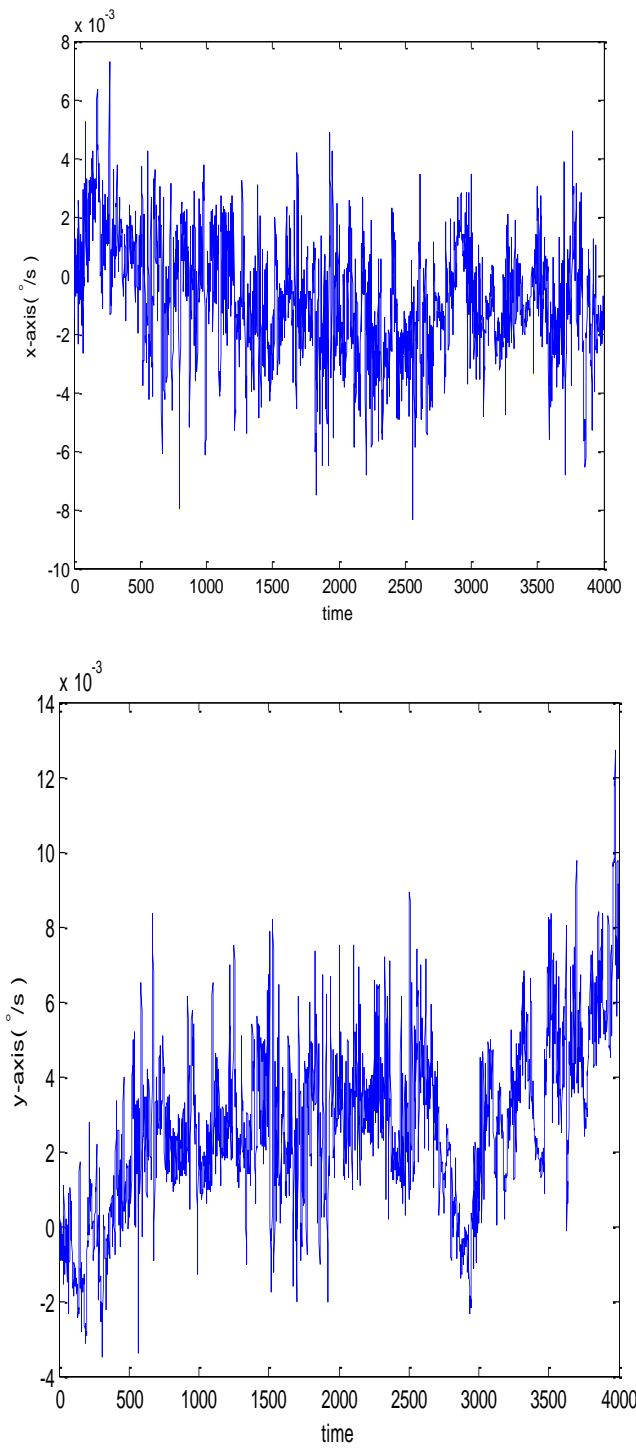

Figure 2. Gyroscope measurements errors. 


\section{SIMULATIONS}

The contribution of errors to the measurement of gyro is simulated with the profile that a gyro is of zero-truth angular velocity and specific force. In this simulation, gyro errors are caused by the bias error and added white measurement noise. The zero-bias of each gyroscope is set as $0.02 \mathrm{deg} / \mathrm{s}$ and the zero-bias of each accelerometer is set as $0.2 \mathrm{~m} / \mathrm{s} 2$. A measurement noise standard deviation of 0.0003 and 0.002 was used for the gyroscopes and the accelerometers, respectively. The gyroscopes' measurements errors are shown in Fig. 2.

The experimental setup is shown in Fig. 3. The performance of the denoised algorithm in FOG static condition can be measured before and after denoising the signal by different metrics such as Allan variance, bias drift and signal to noise ratio.

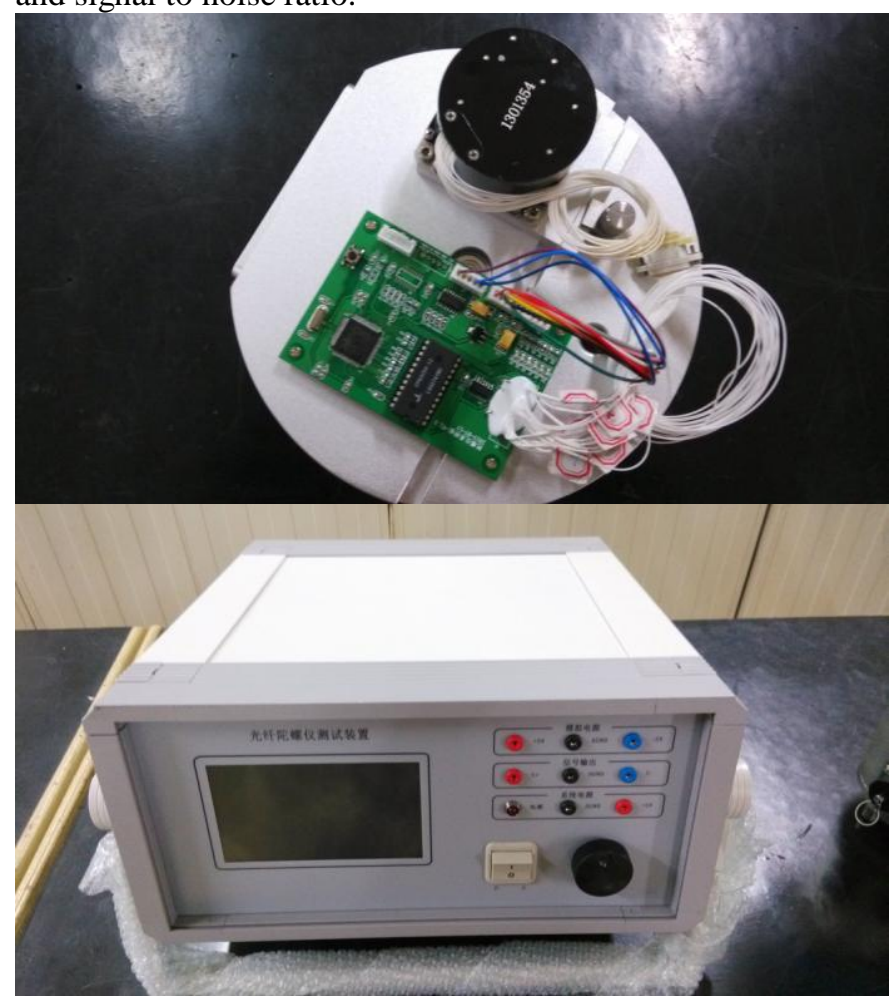

Figure 3. The experimental FOG and its signal processing device.

\section{CONCLUSIONS}

Hybrid Kalman filter algorithm with fading factors is presented in this paper. It uses the innovation sequence to compute fading factors to scale the predicted covariance matrix. The new algorithm thoroughly considers both the optimum and convergence of the filter at the same time. The implementation process of the new algorithm is derived in detail, and it has been tested through computer simulation and practical experiment.

\section{REFERENCES}

[1] S. Sabat, N. Giribabu, J. Nayak, K. Krishnaprasad, Characterization of fiber optics gyro and noise compensation using discrete wavelet transform, in: Proceedings of the 2nd International Conference on Emerging Trends in Engineering and Technology (ICETET), 2009, 909-913.

[2] C. Kownacki, Optimization approach to adapt Kalman filters for the real-time application of accelerometer and gyroscope signals filtering, Digit. Signal Process. 21 (1) (2011) 131-140.

[3] D.W. Allan, J.A. Barnes, A modified Allan variance with increased oscillator characterization ability, in: Proceedings of the 35th Annual Frequency Control Symposium, Baltimore, MD, USA, 1981, pp. 470-475.

[4] Y. Li, Y. Liu, B. Su, Y. Jiang, Modified wavelet filtering algorithm applied to gyro servo technology for the improvement of test-precision, J. Syst. Eng. Electron.22 (3) (2011) 488-492.

[5] H. Chang, L. Xue, W. Qin, G. Yuan, W. Yuan, An integrated MEMS gyroscope array with higher accuracy output, Sensors 8 (4) (2008) 2886-2899.

[6] Rangababu Peesapati, Samrat L. Sabat, K.P. Karthik, J. Nayak, N. Giribabu, Efficient hybrid Kalman filter for denoising fiber optic gyroscope signal, Optik 124 (2013) 4549 - 4556.

[7] I. Zurbenko, P.S. Porter, R. Gui, S. Rao, J. Ku, R. Eskridge, Detecting discontinuities in time series of upper-air data: development and demonstration of an adaptive filter technique, J. Clim. 9 (12) (1996) 3548-3560.

[8] J. Chauvin, N. Petit and P. Rouchon. Kalman filtering for real-time individual cylinder air fuel ration observer on a diesel engine test bench. Technical Report A/340, Ecole des Mines de Paris, 2006.

[9] GAO Weixi, MIAO Lingjuana, NI Maolin. Multiple Fading Factors Kalman Filter for SINS Static Alignment Application. Chinese Journal of Aeronautics 2011;24(1): 476-483.

[10] Sorenson H W, Sacks J E. Recursive fading memory filtering. Information Sciences 1971; 3(2): 101-119.

[11] Ydstie B E, Co T. Recursive estimation with adaptive divergence control. IEE Proceedings D Control Theory and Applications 1985; 132(3): 124-130.

[12] Qin Y Y. Inertial navigation. Beijing: Science Press, 2006. [in Chinese]

[13] Sorenson H W, Sacks J E. Recursive fading memory filtering. Information Sciences 1971; 3(2): 101-119. 